Proceedings

\title{
Servo-Assisted Position-Feedback MEMS Force Sensor with Tunable Sensitivity and Sub-Nanonewton Range ${ }^{\dagger}$
}

\author{
Alessandro Nastro ${ }^{1, *}$, Marco Ferrari ${ }^{1}$, Alfio-Lip Russo ${ }^{2}$, Raffaele Ardito ${ }^{3}$ and Vittorio Ferrari ${ }^{1}$ \\ 1 Department of Information Engineering, University of Brescia, Brescia, Italy; marco.ferrari@unibs.it (M.F.); \\ vittorio.ferrari@unibs.it (V.F.) \\ 2 STMicroelectronics, Italy; alfio-lip.russo@st.com \\ 3 Department of Civil and Environmental Engineering, Politecnico di Milano, Milano, Italy; \\ raffaele.ardito@polimi.it \\ * Correspondence: a.nastro002@unibs.it; Tel.: +39-30-371-5938 \\ + Presented at the Eurosensors 2017 Conference, Paris, France, 3-6 September 2017.
}

Published: 28 August 2017

\begin{abstract}
A Micro Electro-Mechanical System (MEMS) that allows to measure an external force applied to a probe tip exploiting an electrical servo-assisted mechanism based on position feedback is presented. The sensor architecture keeps the position of the probe tip fixed by driving a pair of variable-area electrostatic actuators in a feedback loop controlled by a variable-gap capacitive sensor. By adjusting specific loop parameters, the force sensitivity can be finely tuned electrically. Sub-nanonewton measurement range is achieved with high flexibility in setting the tip working point, making the system promising for measuring forces generated by living biological cells.
\end{abstract}

Keywords: servo-assisted force sensor; MEMS; sub-nanonewton range; tunable sensitivity

\section{Introduction}

Forces generated by living cells are indicators of their state of evolution and can be critical regulators of cell adhesion processes [1]. Microsystems for measuring forces and mechanical properties of different materials at micro/nanoscale have gained an important role for probing biological samples in in vitro mechanobiology experiments [2,3]. In particular, the measurement of minute forces without requiring an associated displacement is a critical task. A common drawback in force sensors is the inherent trade-off between the measurement range and sensitivity. Typically, MEMS force sensors do not exhibit the ability to adapt the range and sensitivity depending on the external applied force [4], which instead would be a desirable feature in many applications. The MEMS force sensor, here presented, allows tunable measurement range and sensitivity, exploiting an electrical servo-assisted mechanism with position-feedback [5] to keep the mechanical structure in a fixed position $x_{0}$ thus allowing to measure an applied external force $F_{\text {ext }}$ offering a virtually infinite mechanical impedance. The device description and operating principle are illustrated in Section 2, experimental results are presented in Section 3, and conclusions are given in Section 4.

\section{Servo-Assisted Position-Feedback MEMS Force Sensor}

The block diagram of the system is shown in Figure 1, while images of the fabricated MEMS are shown in Figure 2a. The input voltage $V_{\text {range }}$ sets the range and sensitivity of force measurement, while the input voltage $V_{\text {pos }_{0}}$ sets the position $x_{0}$ that represents the working point of the probe tip.

The static equilibrium of all forces acting on the shuttle $\mathrm{R}$ integral with the tip, without an applied external force $F_{\text {ext }}$, leads to: 


$$
F_{\text {sens }}-F_{\mathrm{k}}+F_{\mathrm{D} 2}+F_{\mathrm{D} 4}=0
$$

where $F_{\mathrm{D} 2}=\alpha_{\mathrm{D} 2} V_{\mathrm{D} 2}^{2}$ and $F_{\mathrm{D} 4}=\alpha_{\mathrm{D} 4} V_{\mathrm{D} 4}^{2}$ represent the electrostatic forces generated by the variablearea driving actuators $\mathrm{D} 2$ and $\mathrm{D} 4 ; \alpha_{\mathrm{D} 2}$ and $\alpha_{\mathrm{D} 4}$ represent the electro-mechanical transduction factors of the two drivers, respectively [2]. The contribution $F_{\mathrm{k}}=k_{\mathrm{m}} x$ represents the elastic force of the suspending springs, while $F_{\text {sens }}$ is the electrostatic force generated by the voltage $v_{\mathrm{SR}}(\mathrm{t})$ required to measure the variable-gap comb sensing capacitance $C_{S R}$ employing a transimpedance amplifier (OPA656) and a lock-in amplifier (Stanford SR850). The sensing voltage $v_{\text {sens }}(t)=$ $V_{\text {sens }} \sin \left(2 \pi f_{\text {sens }} \mathrm{t}\right)$, with $V_{\text {sens }}=1 \mathrm{~V}_{\text {peak, }}$, is internally downscaled by $\mathrm{A}=0.1$ to set $V_{\text {SR }}$ to $0.1 \mathrm{~V}_{\text {peak, }}$, so to prevent pull-in. The sensing frequency $f_{\text {sens }}$ is set to $100 \mathrm{kHz}$ far from the resonant mechanical frequency at around $2 \mathrm{kHz}$.

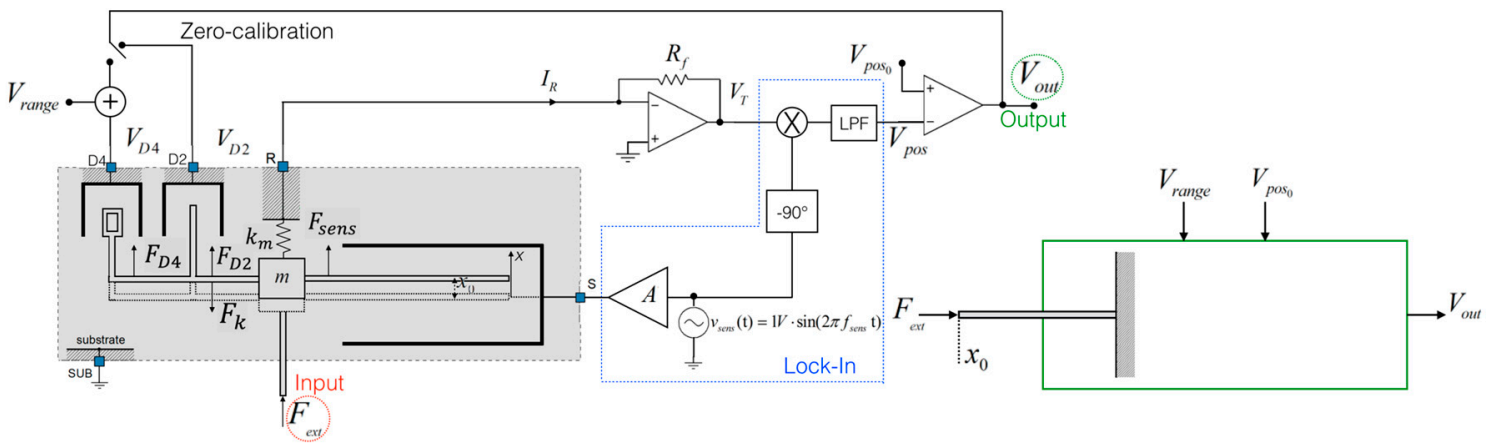

Figure 1. Block diagram of the servo-assisted position-feedback MEMS force sensor.

The initial zero-calibration procedure nulls the output voltage $V_{\text {out }}$ thanks to the zerocalibration feedback loop that modulates the voltage $V_{\mathrm{D} 2}$.

The feedback loop imposes $V_{\text {pos }}=V_{\text {pos }_{0}}$, where $V_{\text {pos }}$ is the output voltage of the transimpedance amplifier which, thanks to the lock-in amplifier, it results linearly dependent to the sensing capacitance $C_{\mathrm{SR}}$ and related to the position $x$ as follows:

$$
V_{\text {pos }}=\mathrm{k} V_{\text {sens }} V_{\mathrm{SR}} \text { A } R_{\mathrm{f}} 2 \pi f_{\text {sens }} C_{\mathrm{SR}}(x)
$$

where $R_{\mathrm{f}}=100 \mathrm{k} \Omega$ is the feedback resistance of the transimpedance amplifier and $\mathrm{k}=1 \mathrm{~V}^{-1}$ is the scale factor of the lock-in amplifier. This procedure allows to select the required voltage $V_{\text {range, }}$ and thereafter, to set the required working point $x_{0}$ by adjusting the voltage $V_{\text {pos }_{0}}$. This ensures that with $F_{\text {ext }}=0$, given the required range and sensitivity, the probe tip is fixed at a desired position $x_{0}$ resulting in $V_{\text {out }}=0$.

Once the zero-calibration procedure has ended, the voltage $V_{\mathrm{D} 2}$ is kept constant, and the feedback loop is switched to the driver D4 in order to measure the external force $F_{\text {ext }}$. The positionfeedback loop keeps the probe tip fixed at the working position $x_{0}$ regardless of the applied force $F_{\text {ext }}$, producing an equivalent infinite mechanical impedance, i.e. unlimited stiffness. Considering the equilibrium of all forces acting on the device, including the external force, results in:

$$
V_{\text {out }}^{2}+2 V_{\text {out }} V_{\text {range }}=F_{\text {ext }} / \alpha_{\text {D } 4}
$$

Linearizing around the working point, it follows that:

$$
V_{\text {out }}=S F_{\text {ext }}
$$

where $S=\frac{1}{2 \alpha_{\mathrm{D} 4} V_{\text {range }}}$ is the force sensitivity of the system.

Considering a non-linearity error of $1 \%$ of the span, the corresponding measurement range is $-0.04 \alpha_{\mathrm{D} 4} V_{\text {range }}^{2}<F_{\text {ext }}<0.04 \alpha_{\mathrm{D} 4} V_{\text {range }}^{2}$. In such a range the output voltage is linearly related to the applied force within an error of $1 \%$, while the measurement range and sensitivity depend only on the voltage $V_{\text {range }}$. 


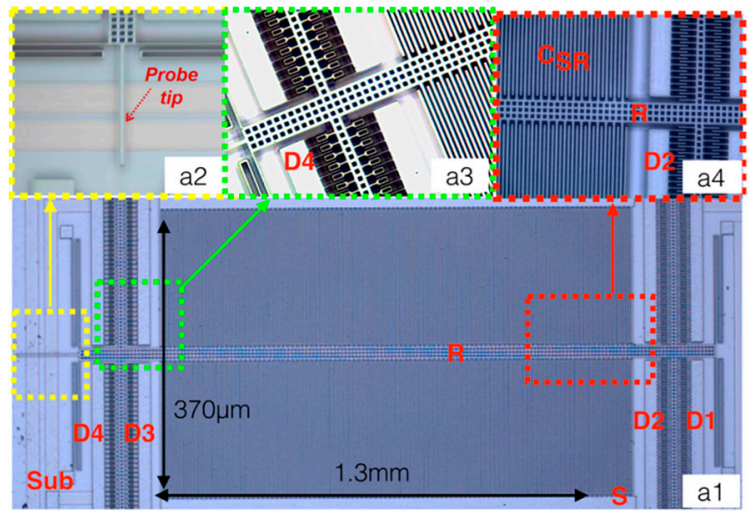

(a)

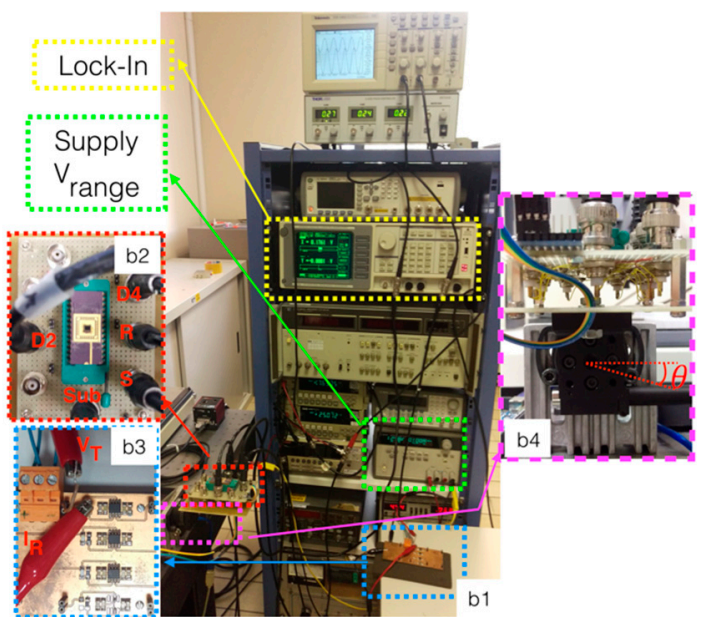

(b)

Figure 2. (a1) Image of the fabricated MEMS device with (a2) enlarged views of the probe tip; (a3) driver D4; (a4) driver D2 and capacitive position sensor $C_{S R}$; (b1) Experimental setup with (b2) enlarged views of the force sensor under test; (b3) the transimpedence amplifier and (b4) the rotating stage.

\section{Experimental Results}

Figure $2 \mathrm{~b}$ shows the setup used to test the device. The weight force controlled by inclination through a motorized ThorLabs MDT693B rotating stage was used as the external reference force. This results in $F_{\text {ext }}=\mathrm{mg} \sin (\theta)$ where $m=24 \mu \mathrm{g}$ is the mass of the movable shuttle, $g=9.81 \mathrm{~m} / \mathrm{s}^{2}$ is the gravitational acceleration and $\theta$ is the inclination angle. Figure $3 a, b$ show the measured output voltage $V_{\text {out }}$ as a function of angle $\theta$ and external force $F_{\text {ext }}$, respectively. In Figure $4 \mathrm{a}$ it is represented the sum $V_{\text {out }}^{2}+2 V_{\text {out }} V_{\text {range }}$ as a function of $F_{\text {ext }}$ from which it is possible to estimate $\alpha_{\mathrm{D} 4}$. Figure $4 \mathrm{~b}$ reports the sensitivity $S$ and the transduction factor $\alpha_{\mathrm{D} 4}$ derived from fitting of Figure 4 a as function of the input voltage $V_{\text {range }}$. Experimental results are in good agreement with the theoretical values of $S$ obtained using the average value of $\alpha_{D 4}$. The inset of Figure $4 \mathrm{~b}$ shows the measurement range of the external forces considering a non-linearity error lower than 1\%, $2 \%$ and $3 \%$. Incremental angles corresponding to $0.4 \mathrm{nN}$ forces where consistently detected, leading to a sub-nanonewton resolution.

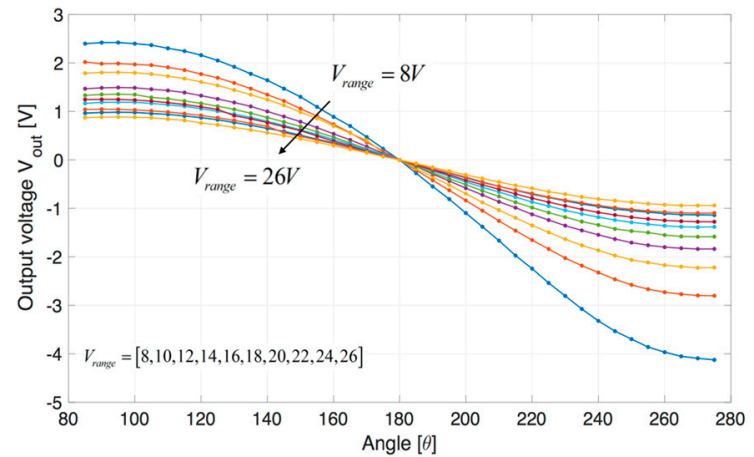

(a)

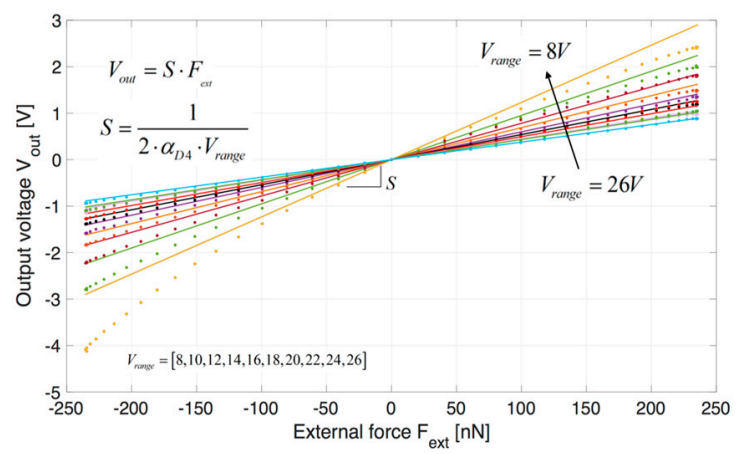

(b)

Figure 3. (a) Measured output voltage $V_{\text {out }}$ as a function of the angle $\theta$ of the rotating stage for different values of $V_{\text {range; }}$ (b) Measured output voltage $V_{\text {out }}$ as a function of the external weight force for different values of $V_{\text {range }}$. Raw data are represented with dots while lines represent a fitting with a polynomial of 1 st order. 


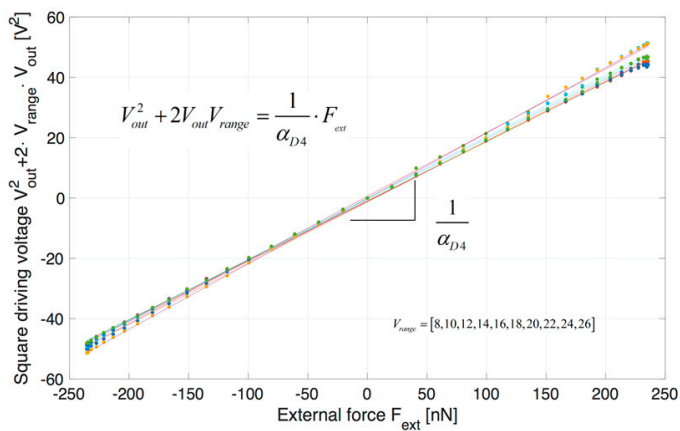

(a)

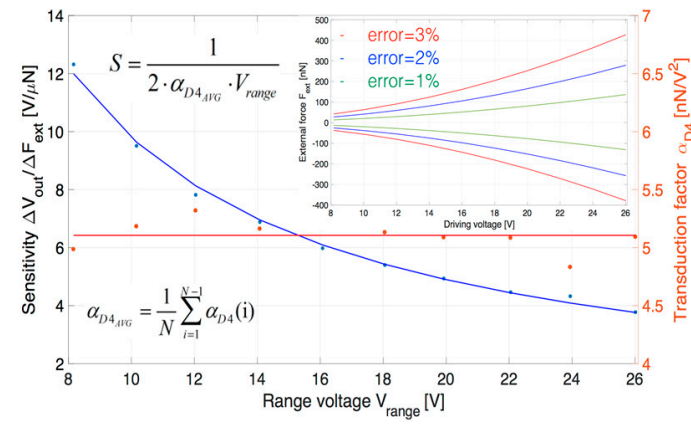

(b)

Figure 4. (a) The estimation of the electro-mechanical transduction factor $\alpha_{\mathrm{D} 4} ;$ (b) Sensitivity $S$ and electro-mechanical transduction factor $\alpha_{\mathrm{D} 4}$ as function of $V_{\text {range }}$. Red line represents the average value of $\alpha_{D 4}$ while blue line is the calculated sensitivity. The inset of Figure $4 \mathrm{~b}$ shows the range of measurable external forces with non-linearity error of $1 \%, 2 \%$ and $3 \%$ of the span.

\section{Conclusions}

A MEMS force sensor that allows to measure an external force applied to a probe tip with tunable sensitivity exploiting an electrical servo-assisted mechanism based on position feedback has been presented. The servo-assisted feedback-position mechanism keeps the position of the probe tip fixed by driving a pair of variable-area electrostatic actuators in a feedback loop controlled by a variablegap capacitive sensor offering a virtually infinite mechanical impedance. By adjusting specific loop parameters, the force sensitivity $S$ and the working position $x_{0}$ can be finely tuned electrically. The experimental results show that the proposed system has the capability of measuring forces in the nanonewton to sub-nanonewton range with sensitivity of up to $12 \mathrm{~V} / \mu \mathrm{N}\left(V_{\text {range }}=8 \mathrm{~V}\right)$ with high flexibility in the setting the tip working point, thereby making the system suitable for biological force probing.

Conflicts of Interest: The authors declare no conflict of interest.

\section{References}

1. Polacheck, W.J.; Chen, C.S. Measuring cell-generated forces: a guide to the available tools. Nat. Methods 2015, 13, 415-423, doi:10.1038/nmeth.3834.

2. Cerini, F.; Ferrari, M.; Ferrari, V.; Russo, A.; Azpeitia Urquia, M.; Ardito, R.; De Masi, B.; Serzanti, M.; Dell’Era, P. MEMS force microactuator with displacement sensing for mechanobiology experiments. AEIT Int. Annu. Conf. 2015, 7415224, 1-6, doi:10.1109/AEIT.2015.7415224.

3. Beyeler, F.; Muntwyler, S.; Nelson, B.J. A six-axis MEMS force-torque sensor with micro-newton and nanoNewtonmeter resolution. J. Microelectromech. Syst. 2009, 18, 433-441, doi:10.1109/JMEMS.2009.2013387.

4. Keekyoung, K.; Ji, C.; Qun, L.; Xiao, Y.W.; Yu, S. Investigation of mechanical properties of soft hydrogel microcapsules in relation to protein delivery using a MEMS force sensor. J. Biomed. Mater. Res. A 2010, 92, 103-113, doi:10.1002/jbm.a.32338.

5. Moore, S.I.; Coskun, M.B.; Alan, T.; Neild, A.; Moheimani, S.O.R. Feedback-Controlled MEMS Force Sensor for Characterization of Microcantilevers. J. Microelectromech. Syst. 2014, 24, 1092-1101, doi:10.1109/JMEMS.2014.2382648. 